\title{
Design and Analysis of 16 Bit Reversible ALU
}

\author{
Lekshmi Viswanath ${ }^{1}$, Ponni.M ${ }^{2}$ \\ (Department of Electronics and Communication, Amrita School of Engineering, Bangalore, India)
}

\begin{abstract}
Reversible or information-lossless circuits have applications in digital signal processing, communication, computer graphics and cryptography. Reversibility plays an important role when energy efficient computations are considered. Reversible logic is used to reduce the power dissipation that occurs in classical circuits by preventing the loss of information. This paper proposes a reversible design of a 16 bit $A L U$. This ALU consists of eight operations, three arithmetic and five logical operations. The arithmetic operations include addition, subtraction, multiplication and the logical operations include NAND, AND, OR, NOT and XOR. All the modules are being designed using the basic reversible gates. The power and delay analysis of the various sub modules is performed and a comparison with the traditional circuits is also carried out.
\end{abstract}

Keywords - Reversible logic circuits, Reversible logic gates, Reversible adder/subtractor, Reversible logic unit, Reversible ALU.

\section{INTRODUCTION}

In modern VLSI system power dissipation is very high due to rapid switching of internal signals. The complexity of VLSI circuits increases with each year due to packing more and more logic elements into smaller volumes. Hence power dissipation has become the main area of concern in VLSI design. Reversible logic has its basics from thermodynamics of information processing. According to this, traditional irreversible circuits generate heat due to the loss of information during computation. In order to avoid this information loss the conventional circuits are modeled using reversible logic. Landauer [1961] showed that the circuits designed using irreversible elements dissipate heat due to the loss of information bits [1]. It is proved that the loss of one bit of information results in dissipation of $\mathrm{KT}^{*} \log 2$ joules of heat energy where $\mathrm{K}$ is the Boltzmann constant and $\mathrm{T}$ is the temperature at which the operation is performed. Benett [1973] showed that this heat dissipation due to information loss can be avoided if the circuit is designed using reversible logic gates [2]. A gate is considered to be reversible only if for each and every input there is a unique output assignment. Hence there is a one to one mapping between the input and output vectors. A reversible logic gate is an $n$-input, $n$ - output device indicating that it has same number of inputs and outputs. A circuit that is built from reversible gates is known as reversible logic circuit.

In this paper, we design a 16 bit reversible ALU that can perform eight operations simultaneously. The eight operations include addition, subtraction, multiplication, division, AND, OR, NOT and XOR. All the modules are simulated in modelsim SE 6.5 and synthesised using Xilinx ISE 12.2.

\section{REVERSIBLE GATES}

Reversible logic is gaining importance in areas of CMOS design because of its low power dissipation. The traditional gates like AND, OR, XOR are all irreversible gates. Consider the case of traditional AND gate. It consists of two inputs and one output. As a result, one bit is lost each time a computation is carried out. According to the truth table shown in Fig.1, there are three inputs $(1,0),(0,1)$ and $(0,0)$ that corresponds to an output zero. Hence it is not possible to determine a unique input that resulted in the output zero. In order to make a gate reversible additional input and output lines are added so that a one to one mapping exists between the input and output. This prevents the loss of information that is main cause of power dissipation in irreversible circuits. The input that is added to an $\mathrm{m} \mathrm{x} \mathrm{n}$ function to make it reversible is known as constant input (CI). All the outputs of a reversible circuit need not be used in the circuit. Those outputs that are not used in the circuit is called as garbage output (GO). The number of garbage output for a particular reversible gate is not fixed. The two main constraints of reversible logic circuit is

$>$ Fan out not allowed

$>$ Feedbacks or loops not allowed. 


\begin{tabular}{|l|l|l|}
\hline $\mathrm{A}$ & $\mathrm{B}$ & $\mathrm{C}$ \\
\hline 0 & 0 & 0 \\
0 & 1 & 0 \\
1 & 0 & 0 \\
1 & 1 & 1 \\
\hline
\end{tabular}

Fig.1.Truth table of conventional AND gate

Fig.1 shows the truth table of conventional AND gate.In order to make this gate as reversible one input and two outputs are to be added so that it becomes a $3 \times 3$ reversible gate.

The main criteria while designing a reversible logic circuit is the minimization of the above mentioned parameters (CI, GO).

\subsection{Basic Reversible Gates}

Several reversible gates have come out in the recent years. The most basic reversible gate is the Feynman gate and is shown in Fig.2. It is the only $2 \times 2$ reversible gate available and is commonly used for fan out purposes. Consider the input $\mathrm{B}$ as constant. When $\mathrm{B}$ is zero, the gate acts as a copying gate or a buffer where both the output lines contain the input A. When B is one, the complement of A is obtained at the output $\mathrm{Q}$. The $3 \times 3$ reversible gates include Toffoli gate, Fredkin gate, New gate and Peres gate, all of which can be used to realize various Boolean functions. Fredkin gate is shown in Fig.3.The $4 \times 4$ reversible gates include TSG gate, MKG gate, HNG gate, PFAG gate etc. Fig. 4 shows the TSG gate. Some of the $4 \times 4$ gates are designed for implementing some important combinational functions in addition to the basic functions. Most of the above mentioned gates can be used in the design of reversible adders.

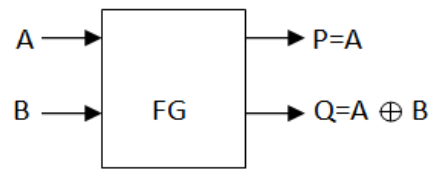

Fig.2. 2x2 Feynman gate

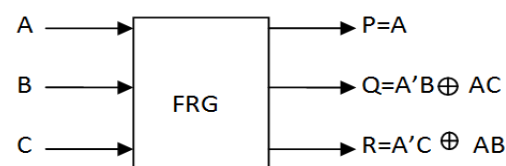

Fig.3. 3x3 Fredkin gate

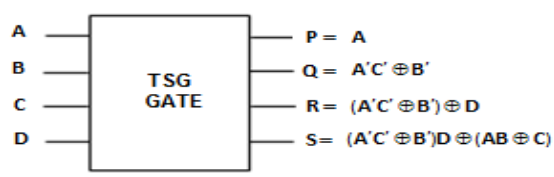

Fig. $4.4 \times 4$ TSG gate

\subsection{Proposed Design}

\section{REVERSIBLE ALU DESIGN}

The Fig.5 shows the basic design of an ALU. For implementing a reversible ALU each of these basic components is implemented using reversible logic. The various sub modules in the design are adder/subtractor, multiplier and a logical unit. All the operations are performed simultaneously. On the basis of control signal, the required result is provided at the output. 


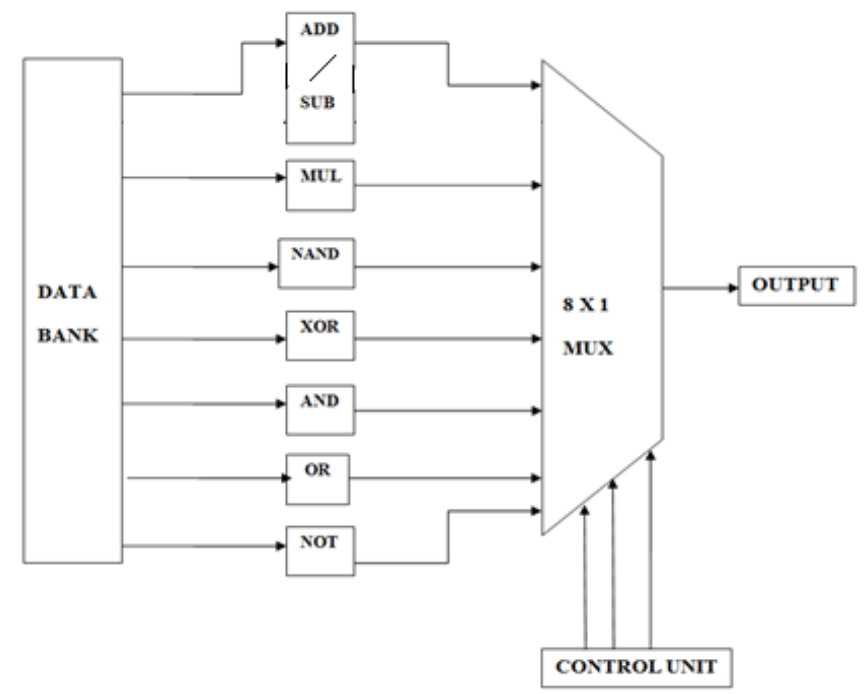

Fig.5. ALU design

\subsection{Bit Adder/Subtractor Design}

The binary full adder/subtractor handles each input along with a carry in /borrow in that is generated as carry out/borrow out from the addition of previous lower order bits. If two $n$ bit binary numbers are to be added or subtracted then $\mathrm{n}$ binary full adder/subtractors should be cascaded. A parallel adder/subtractor is the interconnection of a number of full adder/subtractor and applying the inputs simultaneously. In this paper a 4 bit parallel adder/subtractor circuit is designed using a $4 \times 4$ reversible DKG gate. Fig.6 shows the reversible DKG gate. This gate can acts as an adder or subtractor depending on its control input ' $A$ '. when ' $A$ ' is zero the gate behaves as a full adder and when ' $\mathrm{A}$ ' is one the gate behaves as a subtractor. The block diagramm of a four bit reversible adder/subtractor using DKG gate is shown in Fig.7.

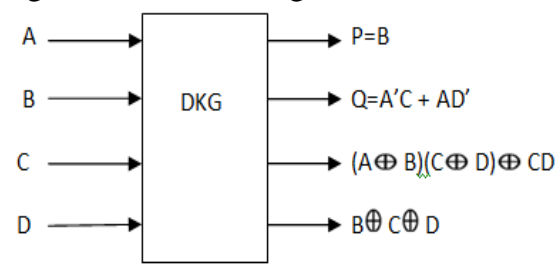

Fig.6. Reversible $4 \times 4$ DKG gate

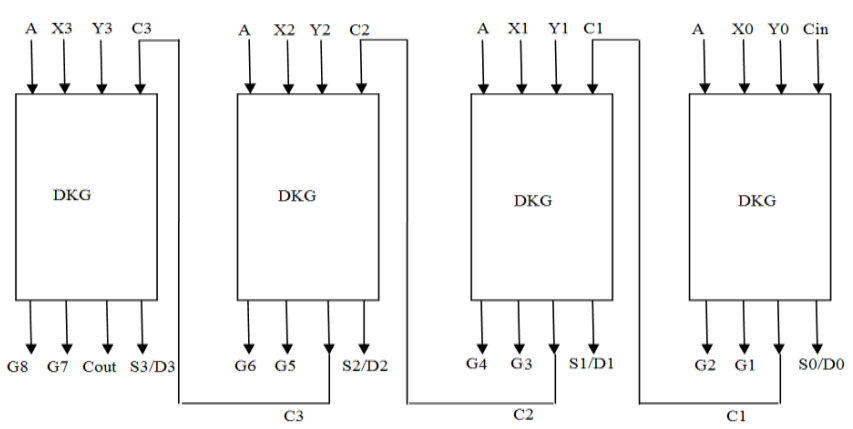

Fig.7. Reversible 4bit adder/subtractor

For implementing a 4bit reversible adder/subtractor, 4 DKG gates are required. Hence the total gate count for a 4 bit adder/subtractor is 4 and that for a 16 bit adder/subtractor is 16 . Here the carry in (Cin) is propagated from one gate to another gate. The module is designed using VHDL, simulated in modelsim and synthesized using xilinx12.2. 


\section{$3.316 \times 16$ Multiplier Design}

In this paper an $\mathrm{n} \times \mathrm{n}$ reversible multiplier is designed using TSG and Fredkin gates. The basic cell for such a multiplier is the full adder block. The TSG gate is shown in Fig.4. It is evident from the figure that the TSG gate can act as full adder when one of the inputs is assigned a constant value of zero [7]. In Fig.4 when $\mathrm{c}$ is assigned as zero the TSG gate acts as a full adder and the sum and carry output is obtained at $\mathrm{S}$ and R respectively. Fig.8 shows the TSG gate implemented as a full adder.

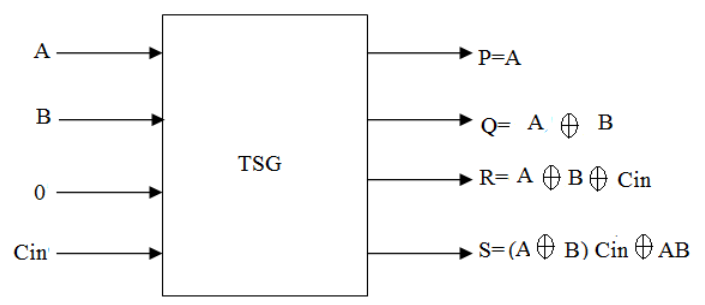

Fig.8. Reversible TSG gate as a full adder

Multiplication includes two basic steps. First step is the generation of partial products and the second one is the addition of the generated partial products. The partial products can be generated using Fredkin gate. When one of the input to the Fredkin gate is assigned a constant value of zero, then the Fredkin gate behaves like an AND gate and partial product can be generated as shown in Fig.10. The generated partial products can now be added using Parallel ripple carry adder that is designed by cascading the TSG gate. For a $4 \mathrm{x} 4$ multiplier, 16 Fredkin gates are required for generating the entire partial products. Three stages of reversible ripple carry adder using TSG gate is required for adding the above generated partial products and obtaining the final 8bit product.

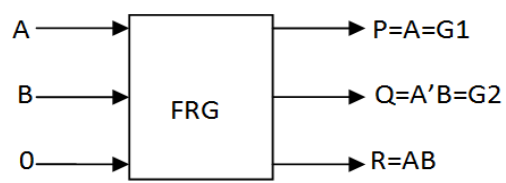

Fig.9 FRG gate used as AND gate.

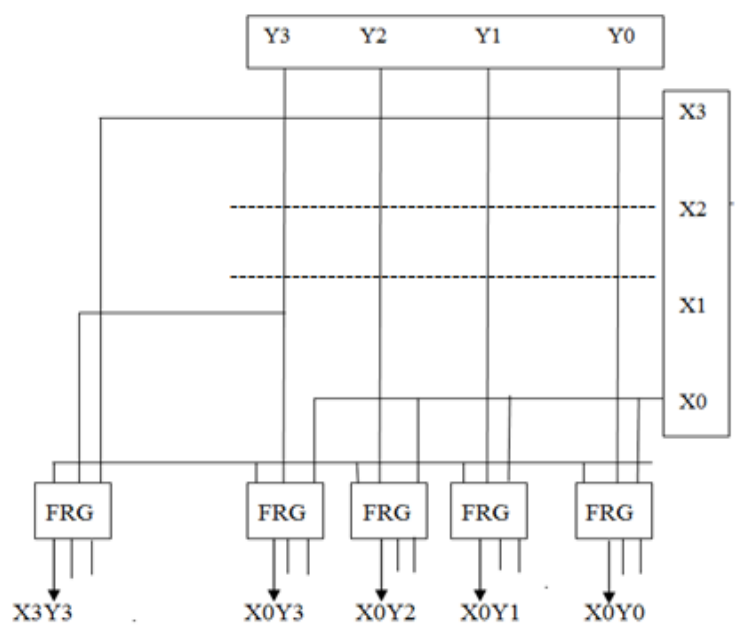

Fig 10. Generation of partial product

\subsubsection{Multiplier Algorithm}

The following algorithm can be used to design an $8 \times 8$ multiplier using $4 \times 4$ multiplier.

1. Let $\mathrm{A}$ and $\mathrm{B}$ be two 8 bit numbers that are to be multiplied.

2. Divide $\mathrm{A}$ into equal halves $\mathrm{A} 1$ and $\mathrm{A} 0$ such that $\mathrm{A} 0$ indicates the $4 \mathrm{bit} \mathrm{LSB}$ and $\mathrm{A} 1$ indicates the $4 \mathrm{bit} \mathrm{MSB}$. Divide B also in the same way as B0 and BI.

3. Multiply A0 and B0 using reversible 4bit multiplier. This forms the first partial product PR1. Retain the www.iosrjournals.org 
4bit LSB of PR1.

4. Now multiply A1 and B0. This forms the partial product PR2. Then multiply A0 and B1. This forms partial product PR3.

5. Add PR2, PR3 and the 4bit MSB of PR1 using reversible parallel adder designed from TSG gate. This forms the temporary result TR1. Retain the 4bit LSB of TR1.

6. Multiply A1 and B1 resulting in the partial product PR4. Now add PR4 and the remaining bits of TR1 (excluding the 4bit LSB) resulting in the temporary result TR2.

7. Now concatenate TR2 with the 4bit LSB of TR1 and PR1. This forms the final 16 bit product. The same can be applied for designing higher multipliers using lower multipliers.

Hence the total gate count for a $4 \times 4$ multiplier is 29 gates (16 Fredkin gates +13 TSG gates).

\subsection{Bit Logical Unit}

In this paper the logical block is designed using PFAG and Feynman gate. For designing a 1bit logical unit one PFAG and one Feynman gate is required. The truth table of PFAG is shown in Fig.12.

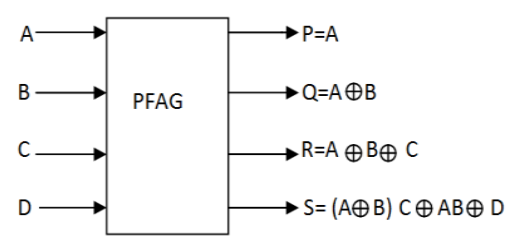

Fig.11 4x4 PFAG gate

Table.1. Truth table of PFAG

\begin{tabular}{|l|l|l|l|l|l|l|l|}
\hline A & B & C & D & P & Q & R & S \\
\hline 0 & 0 & 0 & 0 & 0 & 0 & 0 & 0 \\
\hline 0 & 0 & 0 & 1 & 0 & 0 & 0 & 1 \\
\hline 0 & 0 & 1 & 0 & 0 & 0 & 1 & 0 \\
\hline 0 & 0 & 1 & 1 & 0 & 0 & 1 & 1 \\
\hline 0 & 1 & 0 & 0 & 0 & 1 & 1 & 0 \\
\hline 0 & 1 & 0 & 1 & 0 & 1 & 1 & 1 \\
\hline 0 & 1 & 1 & 0 & 0 & 1 & 0 & 1 \\
\hline 0 & 1 & 1 & 1 & 0 & 1 & 0 & 0 \\
\hline 1 & 0 & 0 & 0 & 1 & 1 & 1 & 0 \\
\hline 1 & 0 & 0 & 1 & 1 & 1 & 1 & 1 \\
\hline 1 & 0 & 1 & 0 & 1 & 1 & 0 & 1 \\
\hline 1 & 0 & 1 & 1 & 1 & 1 & 0 & 0 \\
\hline 1 & 1 & 0 & 0 & 1 & 0 & 0 & 1 \\
\hline 1 & 1 & 0 & 1 & 1 & 0 & 0 & 0 \\
\hline 1 & 1 & 1 & 0 & 1 & 0 & 1 & 1 \\
\hline 1 & 1 & 1 & 1 & 1 & 0 & 1 & 0 \\
\hline
\end{tabular}

In this design the inputs $\mathrm{C}$ and $\mathrm{D}$ is taken as the select lines or control lines.

When $\mathrm{CD}=00$ the PFAG block acts as an AND gate and the output of the AND operation between A and $B$ is obtained at $S$. When $C D=01$ the NAND operation between A and B is performed and the output is obtained at $\mathrm{S}$. When $\mathrm{CD}=10$ the OR operation between $\mathrm{A}$ and $\mathrm{B}$ is performed and obtained at $\mathrm{S}$. The $\mathrm{XNOR}$ operation between $\mathrm{A}$ and $\mathrm{B}$ is obtained at $\mathrm{R}$. When $\mathrm{CD}=11$ the NOT operation of $\mathrm{A}$ is carried out and obtained at $\mathrm{P} 1$. The XOR operation of A and B is obtained at Q irrespective of the value of $\mathrm{C}$ and $\mathrm{D}$. The block diagram of PFAG gate is shown in Fig.11. The design of 1bit logical unit is shown in Fig.12. 


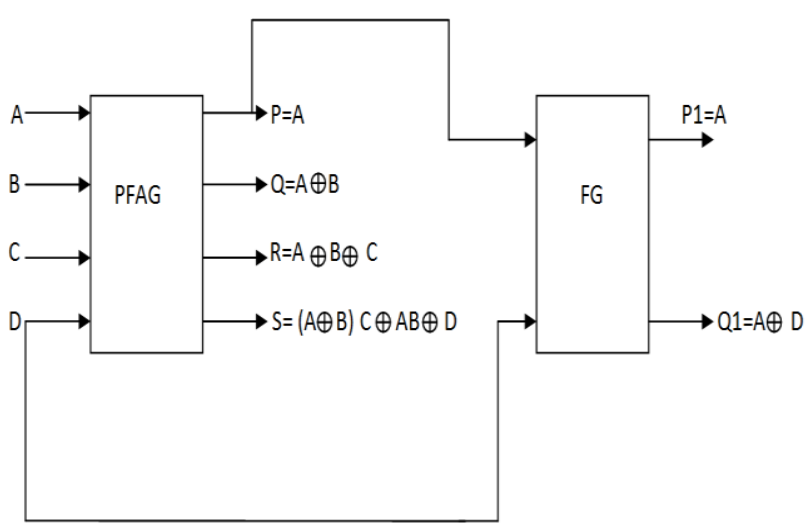

Fig.12. Design of one bit logical unit

The above design requires a total of 32 reversible gates $(16$ PFAG +16 FG) for implementing a 16bit reversible logical unit that can perform AND, OR, NOT,XOR,NAND and NOR.

\section{SIMUlation RESUlts}

All the blocks are modelled using VHDL. The functional verification of the codes is analysed using modelsim 6.5 and synthesised using Xilinx ISE 12.2.

\subsection{Bit Reversible Adder/Subtractor}

Fig. 13 shows the output of 16 bit reversible adder/subtractor. The inputs to this module are the 16bit data ' $A$ ', 'B' and a control signal ' $\mathrm{A} / \mathrm{S}$ '. When the control input is ' 0 ', the addition operation is performed and when the control input is ' 1 ' subtraction operation in carried out. 'Cin' indicates the carry in, Cout indicates the carry out or borrow out obtained from the circuit.

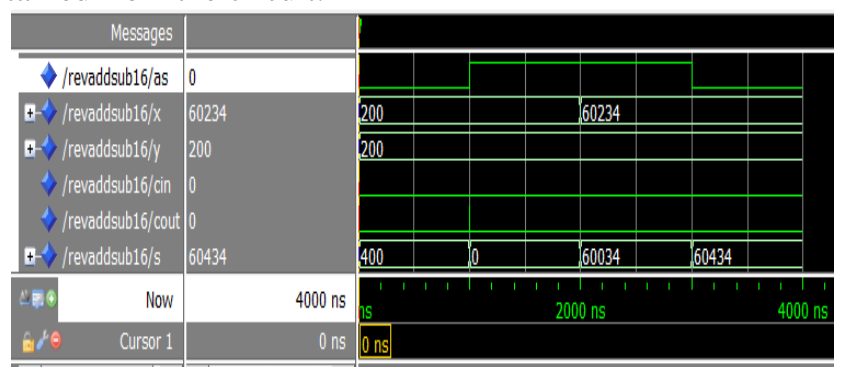

Fig.13. Reversible 16bit adder/subtractor

\subsection{Bit Reversible Logical Unit}

Fig.14 shows the output of reversible 16 bit logical unit. Here A and B indicates the 16bit data. C is a two bit input data that acts as the control signal. Depending on this value the required output results are obtained. AD denotes the AND output, XR denotes the XOR operation, O denotes the OR output and NT denotes the NOT operation.

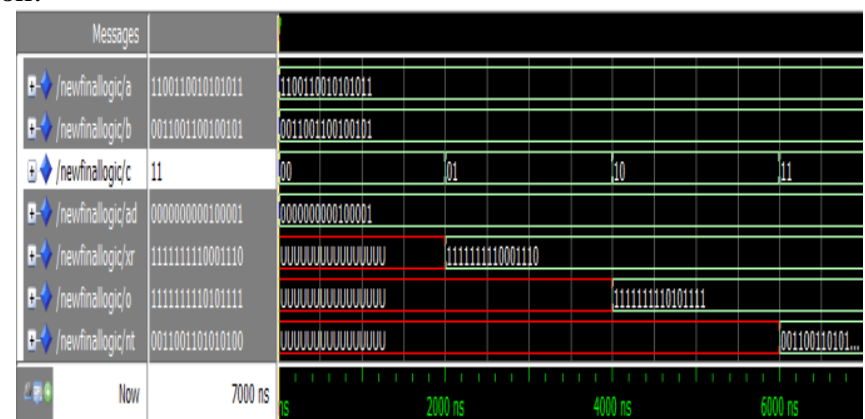

Fig.14. Reversible 16bit logical unit 


\subsection{6 x 16 Multiplier Unit}

Fig. 15 shows the output of $16 \times 16$ reversible multiplier. X and Y indicate the 16 bit input data and PRD indicates the 32bit output data. Here PR1, PR2, PR3 and PR4 are the various partial products that are generated in the multiplication process. All the above mentioned sub modules are integrated together to form a 16 bit ALU.

\begin{tabular}{|c|c|c|c|c|c|}
\hline Messignes & & & & & \\
\hline$\pm \curlyvee /$ reumulititix & 230 & 655535 & & 230 & \\
\hline$\Phi /$ /remultib/y & 65535 & 655255 & & 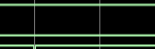 & \\
\hline$t->$ /remulitib/prd & 15073050 & 42498362525 & & 150730050 & \\
\hline$t-\succ /$ remuliti6/pin & 111001010000110 & 111111110000000001 & & 11100101000011010 & \\
\hline 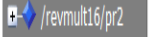 & boco00000000000 & 11111111000000001 & & 1000000000000000000 & \\
\hline t- $/$ /remul|1/16/pr3 & 1110101000010: & 11111111000000001 & & 11100101000011010 & \\
\hline 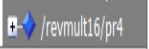 & 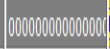 & 1111111000000001 & & 100000000000000000 & \\
\hline 40 & 2000 ps & & 5000 ps & 1000 ps & 1500 ps \\
\hline fire Cussor 1 & $0 p s$ & $0 \mathrm{ps}$ & & & \\
\hline
\end{tabular}

Fig.15. Reversible 16 x 16 multiplier

\section{SYNTHESIS REPORTS}

The delay and power analysis reports of the various submodules are also carried out. The power analysis reports are carried out using synopsys design compiler. The comparison of reversible and conventional submodules in terms of power and delay is evaluated.

\subsection{Reversible Adder/ Subtractor}

\subsection{1 delay analysis}

Table 2 shows the delay analysis of reversible and conventional 4 bit adder and subtractor. It can be deciphered from the figure that reversible adder and subtractor shows $14 \%$ reduction in delay in comparison with conventional adder and subtractor.

\subsubsection{Power analysis}

Table 2. Delay analysis of 4 bit adder and subtractor

\begin{tabular}{|c|c|c|c|c|}
\hline & $\begin{array}{l}\text { Ripple carry } \\
\text { adder }\end{array}$ & Reversible adder & Basic subtractor & Reversible subtractor \\
\hline Delay(ns) & 9 & 7.88 & 9.2 & 7.94 \\
\hline
\end{tabular}

Table 3 shows the power analysis of reversible and conventional adder and subtractor. Reversible adder and subtractor shows $25 \%$ power reduction in comparison with the conventional adder and subtractor.

Table 3. Power analysis of 4 bit adder and subtractor

\begin{tabular}{|l|l|l|c|c|}
\hline & $\begin{array}{l}\text { Ripple carry } \\
\text { adder }\end{array}$ & $\begin{array}{l}\text { Reversible } \\
\text { adder }\end{array}$ & Basic subtractor & Reversible subtractor \\
\hline Power(uW) & 15.2617 & 11.3997 & 16.5745 & 12.3500 \\
\hline
\end{tabular}

\subsection{Reversible Multiplier}

\subsection{1 delay analysis}

Table 4 shows the delay analysis of reversible and conventional multipliers. The reversible multipliers show a better delay in comparison with the traditional multipliers.

Table 4. Delay analysis of reversible and conventional multipliers

\begin{tabular}{|l|l|l|l|l|l|l|}
\hline & $\begin{array}{l}4 x 4 c 0 n . \\
\text { multiplier }\end{array}$ & $\begin{array}{l}4 \times 4 \text { rev. } \\
\text { multiplier }\end{array}$ & $\begin{array}{l}\text { 8x8con. } \\
\text { multiplier }\end{array}$ & $\begin{array}{l}\text { 8x8rev. } \\
\text { multiplier }\end{array}$ & $\begin{array}{l}\text { 16x16con. } \\
\text { multiplier }\end{array}$ & $\begin{array}{l}\text { 16x16rev. } \\
\text { multiplier }\end{array}$ \\
\hline Delay (ns) & 12.760 & 12.644 & 21.524 & 21.440 & 43.198 & 41.178 \\
\hline
\end{tabular}




\subsubsection{Power analysis}

Table 5 shows the power analysis of reversible and conventional multipliers. The reversible and conventional multipliers show $23 \%$ power reduction in comparison with the conventional multipliers.

Table 5. Power analysis of reversible and conventional multipliers

\begin{tabular}{|l|l|l|l|l|l|l|}
\hline & $\begin{array}{l}4 \mathrm{x} 4 \text { con. } \\
\text { multiplier }\end{array}$ & $\begin{array}{l}\text { 4x4rev. } \\
\text { multiplier }\end{array}$ & $\begin{array}{l}\text { 8x8con. } \\
\text { multiplier }\end{array}$ & $\begin{array}{l}\text { 8x8rev. } \\
\text { multiplier }\end{array}$ & $\begin{array}{l}\text { 16x16con. } \\
\text { multiplier }\end{array}$ & $\begin{array}{l}\text { 6x16rev. } \\
\text { multiplier }\end{array}$ \\
\hline Power & $53.256 \mathrm{uW}$ & $36.376 \mathrm{uW}$ & $393.801 \mathrm{uW}$ & $299.868 \mathrm{uW}$ & $2.10 \mathrm{~mW}$ & $1.6 \mathrm{~mW}$ \\
\hline
\end{tabular}

\section{V1. CONCLUSION}

The 16 bit reversible ALU is designed by integrating various sub modules that includes adder/subtractor, multiplier and logical unit. The logical unit performs AND, OR, NOT, XOR, NAND. The performance evaluation of the various submodules are carried out using synopsys tools and it was found that the circuits designed using reversible logic showed a reduced dealy and power. As a future work a reversible divider can also be designed and included into this ALU.

\section{Journal paper}

\section{REFERENCES}

[1] Landauer, R., "Irreversibility and heat generation in the computing process", IBM J. Research and Development, vol. 5 (3): pp. 183191,1961

[2] Bennett, C.H., "Logical reversibility of computation", IBM J. Research and Development, vol. 17: pp. 525-532, 1973

[3] Ravish Aradhya H V, Praveen Kumar B V, Muralidhara K N "Design of Control unit for Low Power ALU Using Reversible Logic" International Journal of Scientific \& Engineering Research Volume 2, Issue 9, September-2011.

[4] B. Raghu Kanth1, B. Murali Krishna2, G. Phani Kumar3, J. Poornima4, K. Siva Rama Krishna "A Comparitive Study Of Reversible Logic Gates”International Journal of VLSI \& Signal Processing Applications, Vol.2,Issue 1, Feb 2012,

\section{Conference papers}

[5] Himanshu Thapliyal ,Nagarajan Ranganathan “A New Reversible Design of BCD Adder” IEEE conference on Design and automation, 2011 pp.1-4.

[6] Zhijin Guan, Wenjuan Li, Weiping Ding, Yueqin Hang, Lihui Ni“An Arithmetic Logic Unit design based on reversible logic gates "IEEE Pacific Rim Conference on Communications, Computers and Signal Processing (PacRim), 2011.

[7] Thapliyal H, Srinivas M.B, "Novel Reversible TSG Gate and Its Application for Designing Components of Primitive Reversible/Quantum ALU," Fifth International Conference on Information, Communications and Signal Processing, 2006.

[8] Madhusmita,SisiraKanta,JagannathSatpathy,MerajSaheeL"Design of Arithmetic Circuits Using Reversible Logic Gates and Power Dissipation Calculation"2010 International Symposium on Electronic System Design, Dec 2010, pp 85-90.

[9] H.Thapliyal,M.B Srinivas "Novel design and reversible logic synthesis of multiplexer based full adder and multipliers" 48th Midwest Symposium on Circuits and Systems, 2005.

[10] Matthew Morrison, Matthew Lewandowski, Richard Meana, Nagarajan Ranganathan, "Design of a novel reversible ALU using an enhanced carry look- ahead adder" 11th IEEE Conference on Nanotechnology (IEEE-NANO), 2011.

\section{Proceedings paper}

[11] J.Bruce, M.A.Thornton, L.Shivakumaraiyah, P.S.Kokate and X.Li "Efficient Adder Circuits Based On a Conservative Reversible Logic Gate" Proceedings of the IEEE Computer Society Annual. 